Cite this as: Chem. Eur. J. 2014, 20, 10439 - 10445

\title{
Characterization of a robust Co(II) fluorescent complex deposited intact on HOPG
}

\author{
María José Heras Ojea, ${ }^{[a]}$ Daniel Reta Mañeru, ${ }^{[a]}$ Lidia Rosado, ${ }^{[a]}$ Juan Rubio Zuazo, ${ }^{[b]}$ German R. \\ Castro, ${ }^{[b]}$ Subrata Tewary, ${ }^{[\mathrm{c}]}$ Gopalan Rajaraman, ${ }^{[\mathrm{c}]}$ Guillem Aromí, ${ }^{[\mathrm{a}]}$ Erika Jiménez, ${ }^{[\mathrm{d}]}$ and E. \\ Carolina Sañudo*[a]
}

\author{
Dedication ((optional))
}

\begin{abstract}
The new di-imine fluorescent ligand ACRI-1 based on a central acridine yellow core is reported along with its coordination complex [Co2(ACRI-1)2] (1), a fluorescent weak ferromagnet. Due to the strong fluorescence of the acridine yellow fluorofore, this is not completely quenched when the ligand is coordinated
\end{abstract}

to $\mathrm{Co}(\mathrm{II})$. The magnetic properties of bulk complex 1 and its stability in solution have been studied. Complex (1) has been deposited on highly ordered pyrolitic graphite (HOGP) from solution. The thin films prepared have been characterized by AFM, ToF-SIMS, GIXRD, XAS, XMCD and theoretical calculations. The data show that the complex is robust and remains intact on the surface of graphite.

Keywords: molecules on surfaces $\bullet$
Cobalt(II) complexes $\bullet$ fluorescent
ligands $\bullet$ magnetic properties $\bullet$

\section{Introduction}

The limit imposed by the superparamagnetic regime present in nanoparticle-based inorganic systems for spintronics sets a miniaturization limit for these systems.[1] At this point, there is a major trend for spintronics: the design of molecular analogues of the inorganic spintronic structures, presenting the possibility of designing cheaper spintronic devices compatible with plastic technology,[2] and the evolution towards molecular spintronics which take advantage of the possibility to tailor molecules with control down to the single spin. Basically, these approaches present the following advantages in front of inorganic spintronics: electron spins can be preserved for longer times and distances, low density, transparency, chemical versatility, flexibility, processability and novel added functionalities.[3] Since the synthesis of polynuclear complexes does not follow a predictable synthetic method, we have to consider the design inside of the

[a] MSc.M. J. Heras-Ojea, MSc. D. Reta Mañeru, L. Rosado, PhD. G. Aromi, PhD. E. C. Sañudo

Departament de Química Inorgànica, Facultat de Química, and Institut de Nanociència i Nanotecnologia IN2UB, Universitat de Barcelona. Av. Diagonal 645 Barcelona 08028 SPAIN

E-mail: esanudo@ub.edu

[b], [d] PhD. J. Rubio Zuazo, PhD. G. R. Castro, PhD. E. Jiménez BM25 SpLine, and ID-08. ESRF, 6 Rue Jules Horowitz, BP 220, 38043 Grenoble, France

[c] Subrata Tewary, PhD.Gopalan Rajaraman

Indian Institute of Technology - Mumbai, INDIA

Supporting information for this article is available on the WWW under http://www.chemeurj.org/ or from the author. serendipitous assembly process. [4] In order to attain multifunctional materials, coordination chemistry molecular systems offer the advantage of the possibility of tailoring ligands to carry desired specific functions, like fluorescence, additional to the magnetic properties provided by transition metal. Additionally, functionalization of the ligands with characteristic chemical groups can also facilitate deposition and organization on surfaces. Thus, different properties can coexist in one same material and be activated by different inputs or there can be synergy between them. The use of multifunctional molecular complexes in the assembly of nanodevices is a hot topic in the fast development of molecular electronics. In order to include these materials in devices and prior any possible application, the study of nanostructuration of the prepared systems is of outmost importance. [5] Molecular magnetism is now progressing in this new track, focusing on the organization of nanomagnets in low-dimensional arrays suitable for addressing single-molecules. Many efforts are being devoted with this goal in mind; however the characterization of thin films, monolayers or sub-monolayers of nanomagnets on surfaces is far from trivial: the amount of material is extremely small and the use of surface sensitive techniques is absolutely necessary. Furthermore, the stability and robustness of the molecular complexes in the deposition conditions must be clearly ascertained. Our group has been interested in nanostructuration of molecular magnets [6] and research efforts are especially being devoted to prepare multifunctional magnetic systems based on molecular species. Gatteschi, Sessoli and co-workers have addressed this issue in several of their publications, where deposition methods are surveyed.[7] Winpenny and co-workers have studied the surface deposition of $\mathrm{Cr}_{7} \mathrm{Ni}$ nanomagnets, [8] with outstanding results. The work on $\mathrm{Mn}_{12}$ and $\mathrm{Fe}_{4}$ single-molecule magnets (SMMs) by Sessoli, Sanctiavit and co-workers has pioneered the use of X-ray absorption (XAS) and X-ray magnetic circular dichroism (XMCD) to characterize thin layers of molecular species on a surface. These studies clearly show that for the $\mathrm{Mn}_{12} \mathrm{SMMs}$ [9] the molecules do not 
remain intact after deposition, usually presenting reduction to $\mathrm{Mn}$ (II) and decomposition, with the loss of the SMM property. The reported data are far better for the $\mathrm{Fe}_{4} \mathrm{SMMs}$, which are robust molecules that can be deposited intact on a surface, maintaining the SMM property.[10] Thus, deposition methods for molecular systems greatly depend on the nature of the molecule and its functional groups. As chemists, the main challenges we face are the design and synthesis of new functional nanomagnets which are robust and can be processed without suffering decomposition. This is the key to being able to use these molecular systems in technological devices in the not-so-far future. In this paper we report our latest results in this direction: The new fluorescent ligand ACRI-1 and its $\mathrm{Co}$ (II) complex [ $\mathrm{Co}_{2}$ (ACRI1)2] (1) are presented. The new fluorescent complex $\mathbf{1}$ has been characterized in bulk and as thin films on a HOPG surface.

\section{Results and Discussion}

We have designed and prepared the fluorescent ligand ACRI-1 based on acridine yellow via Schiff base formation with salicylaldehyde.[11] $]^{1}$ ACRI-1 is a ditopic ligand with two separate coordination pockets which are far enough to avoid quenching of the fluorescence of the central fluorofore by coordination to a metal atom. The fluorescent properties of ACRI-1 and its complexes are derived from the fluorescent group acridine yellow. Reaction of ACRI-1 with $\mathrm{Co}$ (II) nitrate hexahydrate afforded a precipitate which was recrystallized in chloroform to afford dark red crystals of complex $\mathbf{1}$, $\left[\mathrm{Co}_{2}(\mathrm{ACRI}-1)_{2}\right]$ in $25 \%$ yield.

\section{Description of crystal structures}

Table 1 contains the crystallographic data and structural parameters for ACRI-1 and complex 1 (see Supplementary Information for additional crystallographic parameters). The ligand ACRI-1 crystallizes in the Monoclinic space group $\mathrm{C} 2 / \mathrm{c}$ and the crystal structure is shown in Figure 1. The crystal structure shows the intramolecular hydrogen bond between the oxygen atom of the phenol group and the iminic nitrogen. Complex 1 crystallizes in the triclinic space group $\mathrm{P}-1$. The crystal structure and packing diagrams are shown in Figure 2. In $\left[\mathrm{Co}_{2}(\mathrm{ACRI}-1)_{2}\right] \mathbf{1}$ the $\mathrm{Co}(\mathrm{II})$ atoms are tetracoordinated in a distorted tetrahedral environment. The asymmetric unit contains two half molecules ( $\mathrm{Co} 1$ and $\mathrm{Co} 2)$ with a torsion angle $\mathrm{Co} 1-\mathrm{Co} 1^{\prime \cdots} \cdot \mathrm{Co} 2-\mathrm{Co} 2^{\prime}$ of $112^{\circ}$ (Co1' and $\mathrm{Co} 2^{\prime}$ are the symmetry related atoms to $\mathrm{Co} 1$ and $\mathrm{Co} 2$ ), these are shown in green and blue in the packing diagram of Figure 2. The intramolecular Co-Co' distances are Co1--Co1' $12.015 \AA$ and Co2--Co2' $11.901 \AA$. Between different molecules the $\mathrm{Co}(\mathrm{II})-\mathrm{Co}(\mathrm{II})$ distances range between $12.168 \AA$ and $8.069 \AA$. The acridine core of the ligand ACRI1 displays parallel-displaced intramolecular $\pi-\pi$ stacking. The average distance between the aromatic rings is $3.5 \AA$. Between molecules with the same symmetry there are several types of interaction: $\pi-\pi$ stacking between salicylaldehyde rings with average distance of $3.346 \AA, \pi-\pi$ stacking between anthracene and salicylaldehyde rings with average distances of $3.321 \AA$, and H-bonds between the oxygen of the phenoxy group of one molecule with a C$\mathrm{H}$ of salicylaldehyde ring of the neighbour, $\mathrm{O} 2-\mathrm{C} 31^{\prime} \mathrm{C} 31-\mathrm{O} 2$ ' at $3.377 \AA$

Table 1: Crystal data and structure refinement parameters for ACRI-1 and complex 1.

$\begin{array}{lll}\text { Sample } & \text { ACRI-1 } & \text { Complex 1 } \\ \text { Crystal system } & \text { Monoclinic } & \text { Triclinic } \\ \text { Space group } & \text { C2/c } & \text { P-1 } \\ \text { a } / \AA & 27.571(3) & 10.0214(5)\end{array}$

$\begin{array}{lll}\mathrm{b} / \AA & 4.8628(6) & 17.8460(9) \\ \mathrm{c} / \AA & 33.133(4) & 18.2959(9) \\ \alpha /^{\circ} & 90.00 & 104.780(2) \\ \beta /{ }^{\circ} & 101.759(7) & 102.115(2) \\ \gamma /{ }^{\circ} & 90.00 & 106.115(2) \\ \text { Volume } / \AA^{3} & 4348.9(9) & 2897.4(3) \\ Z & 8 & 2 \\ \text { Final R indexes }[\mathrm{I}>2 \sigma(\mathrm{I})] & \mathrm{R}_{1}=0.0634 \mathrm{wR}_{2}=0.1317 & \mathrm{R}_{1}=0.0504 \mathrm{wR}_{2}=0.1487\end{array}$

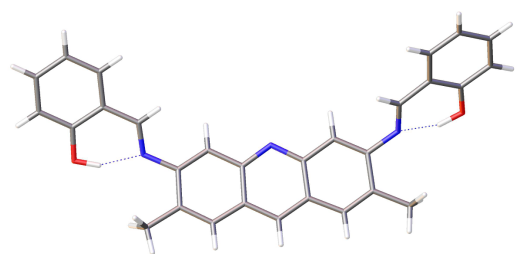

Figure 1. Crystal structure of the ligand ACRI-1. Intramolecular $\mathrm{OH} \cdot \cdot \mathrm{N}$ hydrogen-bonds are shown as dashed lines

a)

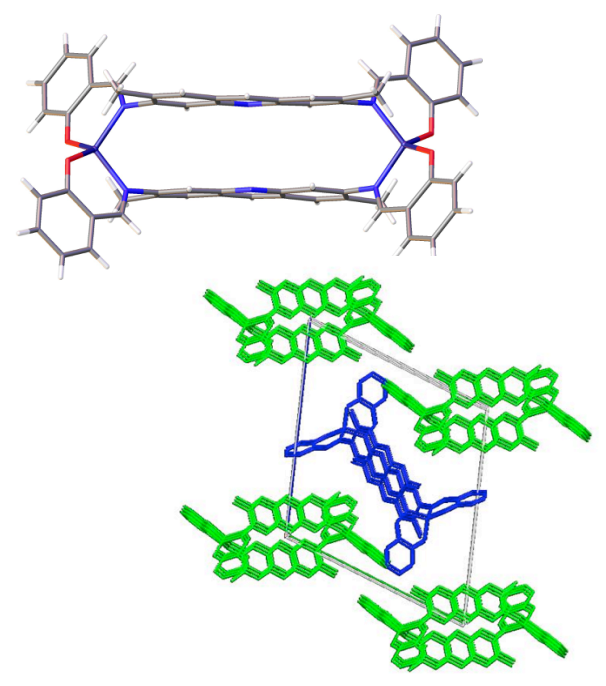

Figure 2. a) Crystal

structure of

one of the two isostructural dinuclear units that compose the asymmetric unit of complex $1 ; b)$ packing diagram where the two crystallographically independent dinuclear units of complex $\mathbf{1}$ are shown in green and blue (colour online).

Between molecules with different symmetry the interactions are $\pi-\pi$ stacking between anthracene rings, and between salicylaldehyde rings with distances between $3.698 \AA$ and $3.309 \AA$. In addition there are Hbonds between the oxygen of the phenoxy group and a $\mathrm{C}-\mathrm{H}$ of the salicyaldehyde ring $\mathrm{O} 3-\mathrm{C} 54 "$ at $3.588 \AA$, and the nitrogen of the anthracene and a C-H of the salicylaldehyde ring N3- 225 " at 3.375 $\AA$. These distances are in agreement with the expected for weak $\mathrm{CH}$ $\mathrm{X}$ hydrogen bonds.[12] The interactions repeat over the crystal packing and even though they are weak by themselves cooperatively they are of vital importance for the magnetic properties of complex $\mathbf{1}$. The intermolecular interactions can be easily visualized calculating the Hirshfield surface,[13] see Additional Information for a figure, which shows that the points of maximum intermolecular interaction, shown in red, are those close to the first coordination sphere of the $\mathrm{Co}(\mathrm{II})$ ions. 


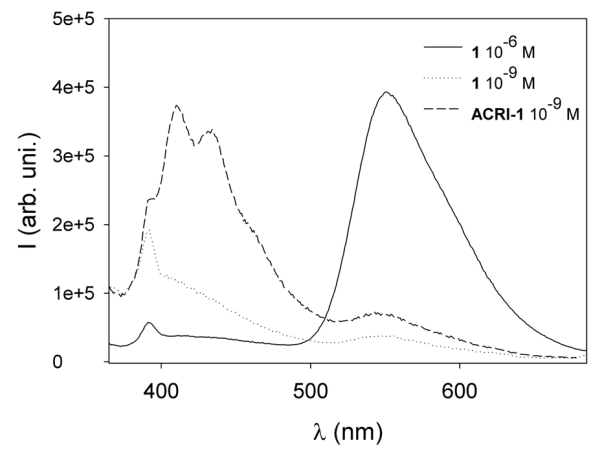
wavelength of $350 \mathrm{~nm}$. The data for complex 1 at $1 \mathrm{e}-6 \mathrm{M}$ has been divided by 20 to fit in the scale.

\section{Fluorescent properties}

Emission spectra of ligand ACRI-1 and complex $\mathbf{1}$ are shown in Figure 3 at an excitation wavelength of $350 \mathrm{~nm}$. In all cases a peak corresponding to excimer formation of the acridine core is observed at circa $550 \mathrm{~nm}$. Dilution to $10^{-9} \mathrm{M}$ of ACRI-1 is necessary to observe the fluorescence of the ligand, which is blue shifted to $425 \mathrm{~nm}$, as usual for anthracene and pyrene derivatives.[14] The emission spectrum of complex 1 shows the expected intramolecular excimer emission around $550 \mathrm{~nm}$ at all concentrations, which should be proves that the molecule remains stable in solution. The coordination of Co(II) does not suppress the fluorescence of the ACRI-1 ligand. However, a quenching of the fluorescence is observed, resulting in less intense signals. The fluorescence spectrum of complex 1 shows no traces of free ligand, supporting the stability of complex $\mathbf{1}$ in solution as observed by NMR (see below).

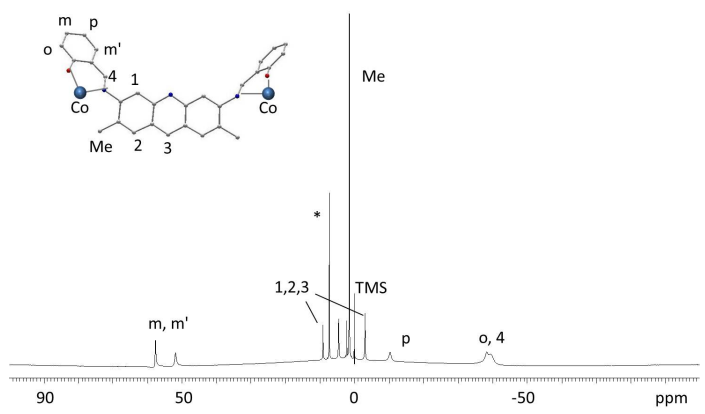

Figure 4. Proton NMR spectrum of complex 1 in deuterated chloroform.

\section{Stability of complex 1 in solution}

In order to deposit complex 1 from solution on a substrate, its stability under the treatment conditions must be established without questions. The solution stability of complex 1 can be assessed by means of mass spectroscopy and paramagnetic proton NMR. The ESI mass spectrum of complex 1 shows the molecular ion peak at $\mathrm{m} / \mathrm{z}=1006$. However, under the conditions of the electrospray, complex 1 breaks-up easily and the most important signals are the peaks for ACRI-1 $(\mathrm{m} / \mathrm{z}=446)$ and acridine yellow from ligand fragmentation $(\mathrm{m} / \mathrm{z}=238)$. A solution of complex 1 in deuterated chloroform was prepared and its proton NMR studied over time. The NMR did not change over long periods of time of up to one day. The proton NMR of solid samples kept at room temperature, open to air, did not change over long periods of time (several months to one year), thus the bulk solid is stable and no oxidation of $\mathrm{Co}(\mathrm{II})$ to $\mathrm{Co}(\mathrm{III})$ due to atmospheric oxygen occurs. The proton NMR of complex 1 is shown in Figure 4, with the signals assigned to the respective protons as indicated. The simple NMR clearly shows that the complex is stable in solution and that the ligand remains coordinated to the complex. The solution can be driven to dryness by evaporation of the $\mathrm{CDCl}_{3}$ and then the solid re-dissolved in deuterated chloroform again: no changes are observed in the spectrum. The proton NMR of the precipitate, as obtained from the reaction, without further treatment, is that of complex $\mathbf{1}$, with a small amount of free ligand. A treatment of this precipitate at $200^{\circ} \mathrm{C}$ for 2 hours in an oven results in the elimination of the free ligand and a residue of pure complex $\mathbf{1}$, as checked by proton NMR. Thus the NMR and mass spectroscopy, together with the fluorescence analysis of complex $\mathbf{1}$ in solution show that it is stable and further processing using a solution of the complex can be pursued.

\section{Bulk magnetic properties}

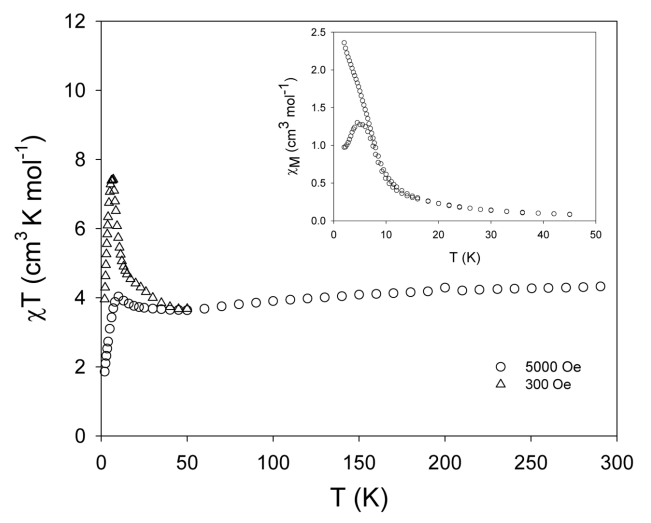

Figure 5. DC magnetic susceptibility data for complex 1 shown as a $\chi \mathrm{T}$ vs. T plot at 5000 and 300 Oe, and zero-field cool/field cool plot at 300 Oe (inset).

The magnetic susceptibility of $\mathbf{1}$ was investigated in the temperature range $2-300 \mathrm{~K}$ at applied fields of 300 and $5000 \mathrm{Oe}$. The plot of $\chi_{\mathrm{M}} T$ vs $T$ is shown in Figure 5. Upon cooling the $\chi_{\mathrm{M}} T$ value is nearly constant down to $60 \mathrm{~K}$. Below this temperature the $\chi_{\mathrm{M}} T$ product increases to $4.06 \mathrm{~cm}^{3} \mathrm{~mol}^{-1} \mathrm{~K}$ at $15 \mathrm{~K}$. Below $15 \mathrm{~K}$ the $\chi_{\mathrm{M}} T$ product drops to a value of $1.85 \mathrm{~cm}^{3} \mathrm{~mol}^{-1} \mathrm{~K}$ at $2 \mathrm{~K}$. The $\chi_{\mathrm{M}} \mathrm{T}$ value $4.31 \mathrm{~cm}^{3}$ $\mathrm{K} \mathrm{mol}^{-1}$ at $300 \mathrm{~K}$ is in agreement with the calculated value of 3.75 $\mathrm{cm}^{3} \mathrm{~K} \mathrm{~mol}^{-1}$ for two magnetically isolated distorted tetrahedral Co(II) atoms $(\mathrm{S}=3 / 2, \mathrm{~g}=2.0)$. Ac magnetic susceptibility data of complex 1 were collected at different frequencies of an oscillating ac field of 4 Oe. A signal is observed in the out-of-phase susceptibility with a maximum at $5 \mathrm{~K}$ indicating the onset of ferromagnetic order. This long range interaction is promoted by the intermolecular interactions in the 3D network of complex $\mathbf{1}$, as discussed before. The peak in the out-of-phase ac magnetic susceptibility has a maximum at $5 \mathrm{~K}$, and the ZFC-FC measurement shows a splitting of the two curves at this same temperature (as shown in the inset of Figure 5) indicating that 5 $\mathrm{K}$ is the blocking temperature for the ferromagnetic order of complex 1. Many molecular magnets based on $\mathrm{Co}(\mathrm{II})$ are reported in the 
literature. Usually, mononuclear tetrahedral Co(II) complexes with weaker intermolecular interactions than those found in complex $\mathbf{1}$ order at temperatures below $2 \mathrm{~K}$,[15] while the tridimensional coordination polymer $\mathrm{Co}(\mathrm{dca}) 2$ has ferromagnetic order at $9 \mathrm{~K}$.[16]

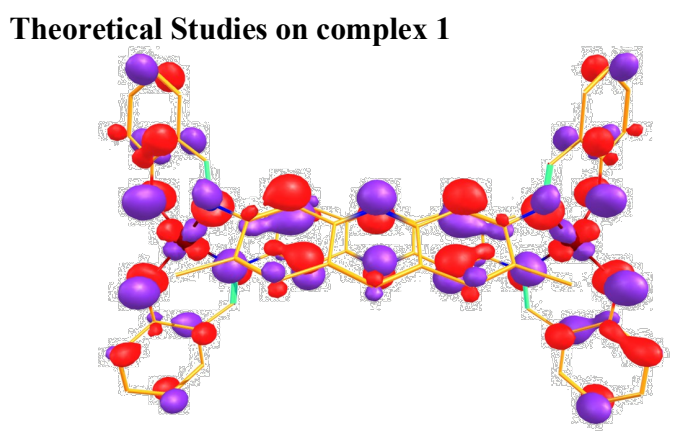

Figure 6. Highest occupied molecular orbital (HOMO) of complex 1.

The X-ray structure was treated as an initial guess to perform the quantum chemical calculations for both intra and inter molecular interactions in complex 1. The DFT [17] calculations using Gaussian 09 suite programs [18] were carried out to yield the single point energies of all possible electronic states (see ESI for details). Single point computed structure of the tetramer has been shown in Figure 6a. The magnetic exchange interactions were calculated for complex 1 to underpin the mode of coupling between the two tetrahedral $\mathrm{Co}$ (II) ions mediated via extended aromatic ring system. DFT calculations reveals that both the intra and inter dimer exchange interactions are ferromagnetic in nature with $J$ value of $0.15 \mathrm{~cm}^{-1}$ and $0.03 \mathrm{~cm}^{-1}$ respectively. The computed values are in good agreement to the experimental observation. The magnitude of intramolecular exchange is relatively weak, essentially due to the long distance between the two Co(II) centres. The interdimer exchange is much weaker as the exchange interactions are mediated via H-bonding interactions. The spin density plots are shown in Figure 6. In all cases the spin density on the $\mathrm{Co}$ (II) atom are ca. 2.7 suggesting significant delocalization of spin density to other atoms. Particularly the coordinated N- and Odonor atoms have significant positive spin density due to spin delocalization. Particularly on coordination nitrogen spin density of $\sim 0.05$ has been detected and this is in accord to the XAS N-edge spectra discussed below. One of the magnetic orbitals (HOMO) of 1 is shown in Figure 6 as the unpaired electrons in $\mathrm{Co}(\mathrm{II})$ are present in $\mathrm{t}_{2}$ set of orbitals possessing $\sigma$-character and this promotes spin delocalization to the coordinating atoms (see ESI for the computed Eigen value plot for complex 1). From the coordinating atoms spin polarization propagates as evidence from the alternating spin densities on the spacer atoms. Significant contributions from ACR-I ligand to the HOMO reveals that observed exchange is superexchange in nature via the aromatic spacer ligands. The ferromagnetic coupling is essentially related to the metal-metal distance and number of the spacer groups/atoms. Earlier prediction of long range coupling on $\mathrm{Cu}$ (II) complex suggest that the exchange should be ferromagnetic in this case as there are three aromatic spacer groups.[19]

Surface deposition and characterization
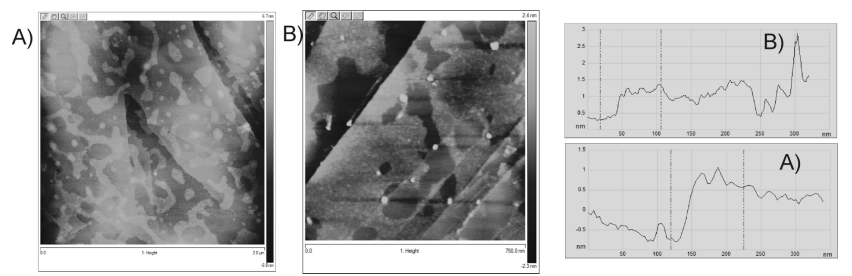

Figure 7. AFM images (A) $2 \mu \mathrm{m}$ image and B) $750 \mathrm{~nm}$ image) and depth profile of two samples of a 1e-5 M solution of complex 1 deposited by spin-coating on HOPG.

To study the surface deposition, a $10^{-5} \mathrm{M}$ solution of complex $\mathbf{1}$ in acetone was deposited onto freshly cleaved HOPG by spin coating at $500 \mathrm{rpm}$ during 30 seconds. Clean acetone was deposited on HOPG by spin coating in the same conditions as those used to prepare the sample of complex $\mathbf{1}$ and the AFM showed a pristine HOPG surface. The topography of the samples was characterized by atomic force microscopy (AFM) using peak force mode. Figure 7 shows two AFM images of a thin-layer of complex 1 with an average height of $1.2 \mathrm{~nm}$. The observed $1.2 \mathrm{~nm}$ height of the layer fits with the height of the molecule when it is placed with the salicylaldehyde groups interacting by $\pi-\pi$ stacking with the HOPG surface. A ToF-SIMS experiment was performed in order to see of complex 1 was deposited and remained intact under the deposition conditions. The surface used was conductive $\mathrm{Si}$, since the ToF-SIMS technique requires a conductive surface. The samples were prepared under the same spincoating conditions used to prepare the samples for AFM, one was studied by TOF-SIMS and the other by AFM. The AFM revealed a continuous coverage of the surface with aggregates with heights of around 1 to $2 \mathrm{~nm}$ distributed on the surface. The TOF-SIMS spectra (see supplementary information) showed peaks at 446 and $488 \mathrm{~m} / \mathrm{z}$ which can be associated to the [(ACRI-1)+H]+ and [Co(ACRI-1)$\mathrm{Me}$ - fragments, and the presence of Cobalt in the deposited layer, confirming that the aggregates observed in the AFM images correspond to molecules of complex $\mathbf{1}$, which had been deposited intact on the surface. Grazing incidence X-Ray diffraction (GIXRD) was performed on a spin coated HOPG sample, prepared in the same conditions as the AFM samples, and compared to that of a pellet of bulk complex 1 . Unfortunately, the diffraction features observed were very weak due to the extremely low quantity of material of the monolayer and the very strong diffraction signals of the polycrystalline HOPG substrate. A saturated solution of complex 1 was drop casted on a glass plate and GIXRD of the thin film measured, it showed a weak diffraction pattern like that of the pellet of bulk complex 1, (see the Supplementary Information). Thus, polycrystalline thin layers of complex 1 can be deposited on a surface. In the GIXRD of the spin coated HOPG, some diffraction peaks observed over long acquisition times coincided with those of the bulk sample of $\mathbf{1}$ and the drop-casted $\mathbf{1}$ on glass. Bulk complex $\mathbf{1}$ and thin films of complex 1 on HOPG were studied by X-ray absorption spectroscopy (XAS) at ESRF beamline ID-08. XAS spectra were taken at both Co- and N-edge. The X-ray magnetic circular dichroism (XMCD) spectra is defined by the difference between the absorption spectra collected with left and right polarized light. XAS spectra and the dichroic signal at Co-edge for a thin layer of complex 1 on HOPG and a powder sample of complex $\mathbf{1}$ are shown in Figure 8. A full study was performed varying the field and temperature down to $10 \mathrm{~K}$, both the powder of complex 1 and the thin layer of complex 1 on HOPG displayed a paramagnetic signal. Unfortunately, $10 \mathrm{~K}$ is above the 
blocking temperature of the ferromagnetic order of complex $\mathbf{1}$, thus we have been unable to establish whether the thin layer of complex $\mathbf{1}$ displays long-range magnetic order as the bulk sample does. Clearly the spectra of complex $\mathbf{1}$ on HOPG is very similar to that of bulk complex $\mathbf{1}$, proving that complex $\mathbf{1}$ is intact after deposition on HOPG by spin coating. A simulation of the spectrum with the software CTM4XAS [20] is also shown in Figure 8. The software performs atomic multiplet calculations and allows the crystal field parameters to be adjusted. The spectral features observed both for complex 1 and complex 1 on HOPG can be simulated with crystal field parameters in agreement with those expected for distorted tetrahedral $\mathrm{Co}$ (II) in a coordination environment like that found in complex $\mathbf{1}$. It has been found that in both cases the spectral are well simulated for $\mathrm{Co}$ (II) in a distorted tetrahedral environment. The crystal field parameters used in the simulation are $10 \mathrm{Dq}$, which is the crystal field splitting of the d-orbitals (in the program a negative value is used for tetrahedral crystal field), Dt and Ds, which reflect the distortion with respect to an ideal environment. The best simulation of XAS and XMCD spectra was obtained for $10 \mathrm{Dq}=0.63 \mathrm{eV}\left(5081 \mathrm{~cm}^{-1}\right), \mathrm{Dt}=0.025$ and Ds $=-0.15$ for the powder complex 1 sample and $10 \mathrm{Dq}=0.70 \mathrm{eV}$ $\left(5645 \mathrm{~cm}^{-1}\right), \mathrm{Dt}=0$ and $\mathrm{Ds}=-0.2$ for the thin layer of complex 1 on HOPG, and these are shown as dashed lines in Figure 8. The 10Dq values obtained are in agreement with the expected 10Dq values for tetrahedral $\mathrm{Co}(\mathrm{II})$ with $\mathrm{N}$ and $\mathrm{O}$ donnors.[21] At N-edge, and XLD signal was measured presenting a maximum at 45 degrees from the plane of the surface, in agreement with the DFT calculations that suggest that there is unpaired electron density on the $\mathrm{N}$ atoms of the ACRI-1 ligand.
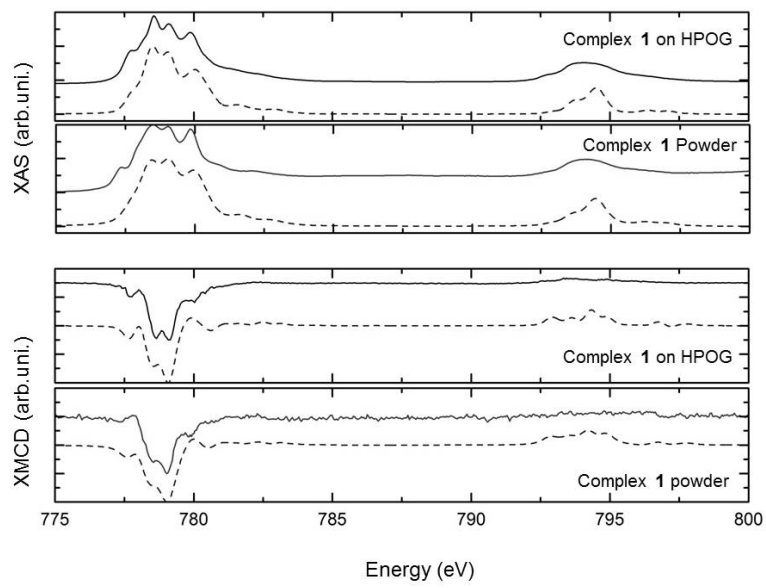

Figure 8. X-ray absorption (XAS) and X-ray magnetic circular dichroism plots for a thin layer on complex 1 deposited on HOPG by spin coating (Complex 1 on HOPG) and a bulk sample of complex 1 (Complex 1 powder). The dashed lines are simulations calculated using CTM4XAS (see text for the simulation parameters).

\section{Conclusion}

We have presented here the new multifunctional material $\left[\mathrm{Co}_{2}(\mathrm{ACRI}-1)_{2}\right]$ (1) with fluorescent and magnetic properties. The stability of the complex has been established and surface deposition studies performed. The fluorescent complex 1 can be deposited intact on a surface, as shown by XAS. The XAS and XMCD spectra of complex 1 can be modeled with crystal field parameters in agreement with the crystal structure of the molecule. The N-edge measurements suggest a preferential orientation of the molecules on the HOPG surface. For possible applications of nanomagnets in devices, this is a key point that still needs to be carefully assessed, especially when the nanomagnets in question are polynuclear coordination complexes.

\section{Experimental Section}

All chemicals were purchased from commercial sources and used as received.

$\mathrm{C}_{29} \mathrm{H}_{23} \mathrm{~N}_{3} \mathrm{O}_{2}$ (ACRI-1). To a mixture of acridine yellow hydrochloride $\left(\mathrm{C}_{15} \mathrm{H}_{16} \mathrm{~N}_{3} \mathrm{Cl}\right)$ $(1.215 \mathrm{~g}, 4.45 \mathrm{mmol})$ and triethylamine $(3.10 \mathrm{~mL}, 22.23 \mathrm{mmol})$ in ethanol $(100 \mathrm{~mL})$ was added salicyladehyde $(931 \mu \mathrm{L}, 8.89 \mathrm{mmol})$ The resulting solution was stirred and heated up to $79{ }^{\circ} \mathrm{C}$. Reflux was maintained during eight hours. After standing overnight, a precipitate was filtered, washed with ethanol and dried with diethyl ether. The yield of the reaction is $73 \%$ ( $1.45 \mathrm{~g}$ obtained). Suitable crystals for single-crystal X-ray diffraction were grown from concentrated acetonitrile solution by slow evaporation. Elemental Analyses: calculated for $\mathrm{C}_{29} \mathrm{H}_{23} \mathrm{~N}_{3} \mathrm{O}_{2} \cdot 1 / 2 \mathrm{H}_{2} \mathrm{O}$ : C, $76.62 \% \mathrm{H}, 5.32 ; \mathrm{N}, 9.24 \%$; Experimental: C, 76.6; H, 5.06; N, 9.14\%. Selected IR data in $\mathrm{cm}^{-1}(\mathrm{KBr}): 3310.72(\mathrm{~m})$, $3196.04(\mathrm{~s}), 1645.65(\mathrm{~s}), 1569.05(\mathrm{~m}), 1504.37(\mathrm{~s}), 1460.63(\mathrm{~m}), 1388.36(\mathrm{~m}), 1353.19$ (m), $1185.98(\mathrm{~m}), 1130.18(\mathrm{~m}), 847.79(\mathrm{~m}), 759.94(\mathrm{w}), 635.00(\mathrm{w})$.

[Co2(ACRI-1) 2 (1). A solution of the ligand ACRI-1 $\left(\mathrm{C}_{29} \mathrm{O}_{2} \mathrm{~N}_{3} \mathrm{H}_{21}\right)(0.25 \mathrm{~g}, 0.56 \mathrm{mmol})$ in acetonitrile $(20 \mathrm{~mL})$, was treated with $\mathrm{Co}\left(\mathrm{NO}_{3}\right)_{2} .6 \mathrm{H}_{2} \mathrm{O}(0.16 \mathrm{~g}, 0.56 \mathrm{mmol})$ and three equivalents of $\mathrm{Et}_{3} \mathrm{~N}(240 \mu \mathrm{L}, 1.68 \mathrm{mmol})$. After 24 hours of stirring a precipitate was filtered, washed with acetonitrile, and dried with anhydrous diethyl ether. Yield $25 \%$ $(0.14 \mathrm{~g})$. Slow diffusion of hexanes into a chloroform solution of the precipitate results in the formation of single crystals of $\left[\mathrm{Co}_{2}(\mathrm{ACRI}-1)_{2}\right]$ (1) suitable for X-ray diffraction. MW $=1006 \mathrm{~g} / \mathrm{mol}$. ESI-MS Ms $/ \mathrm{z}=1006$. Elemental analysis calculated (experimental) for $\mathrm{C}_{66} \mathrm{H}_{72} \mathrm{Co}_{2} \mathrm{~N}_{10} \mathrm{O}_{13}$ : C 59.53 (59.79), H 5.45 (4.78), N 10.52 (10.20) \%. Selected IR data in $\mathrm{cm}^{-1}(\mathrm{KBr}): 3442.51(\mathrm{~m}), 3007.80(\mathrm{~s}), 1605.03(\mathrm{~s}), 1575.30(\mathrm{~s}), 1528.98(\mathrm{~s}), 151494.80$ $(\mathrm{m}), 1456.99(\mathrm{~s}), 1435.93(\mathrm{~s}), 1383.90(\mathrm{~m}), 1321.39(\mathrm{~m}), 1276.09(\mathrm{~m}), 1184.57(\mathrm{~m})$, $1151.53(\mathrm{~m}), 1131.64(\mathrm{~s}), 915.23(\mathrm{~m}), 869.03(\mathrm{~m}), 752.90(\mathrm{~s}), 635.49(\mathrm{~m}), 577.47(\mathrm{w})$.

Single crystal diffraction data were collected on a Bruker APEXII SMART diffractometer at the Facultat de Química, Universitat de Barcelona, using a microfocus Molybdenum k $\alpha$ radiation source. The structures were solved by direct methods (SHELXS) and refined on $\mathrm{F}^{2}$ (SHELX). CIF files for the reported complexes have been deposited at the Cambridge Crystallographic Data Centre and allocated with deposition numbers CCDC 917646 (ACRI-1) and 917647 (Complex 1) and are available upon request at the website: http://www.ccdc.cam.ac.uk/. IR, UV-vis and fluorescence spectra were recorded at the in-house facilities. Elemental analyses, magnetic measurements (SQUID magnetometer equipped with a 5T magnet on crushed crystals) and ${ }^{1} \mathrm{H}-\mathrm{NMR}$ (Varian Unity $300 \mathrm{MHz}$ ) were performed at the CCiTUB. Surface characterization was done at the CCiT-UB by using an AFM Multi mode. The electronics of the system are Nanoscope V from Bruker. Topographic mode: peak force. Each image has a resolution of $512 \times 512$ pixels, taken at a velocity of 1 row/s. Tip used: silicon oxide with a nominal radius of $5 \mathrm{~nm}$. The X-ray diffraction experiment at grazing incidence angle was carried out at a fixed wavelength of $0.826 \AA$ on a six-circle diffractometer, using a 2D-charge coupled device (Photonic Science CCD) detector, at SpLine beamline (BM25B), ESRF, Grenoble, France. The detector has an active area of $250 \times 125 \mathrm{~mm}^{2}$ and $603825 \times 1913$ (2x2 binned) pixels, with a 65.6 micron pixel size. The sample-detector distance during the experiment varied from 180 up to $630 \mathrm{~mm}$. The X-ray magnetic circular dichroism (XMCD) measurements have been performed at the beamline ID08 at ESRF, which is based on Apple II undulator source allowing a full control of linear and circular polarization and a high scanning speed spherical grating monochromator Dragon type. The absorption signal has been measured in total electron yield at $10 \mathrm{~K}$. A magnetic field parallel to the incident beam was provided by a superconducting electromagnet. Computational details: Magnetic exchange coupling constant $(J)$ was evaluated by employing the Noodleman's broken symmetry approach, as developed by Ginsberg and Noodleman.[22] The hybrid B3LYP functional [23] which is known to better reproduce the numerical estimates of magnetic exchange especially for transition metal complexes have been used for our calculations. Ahlrichs triple- $\zeta$ valence basis set (TZV)[24] employed over all the elements in order to have better understanding in terms of the energies and spin densities. Molekel version 4.3 has been used to generate the spin density plots and Chemcraft version 1.6 has been used to visualize and represent the geometry of the complexes for this article.[25] For calculating magnetic susceptibility along with the relative spin state energies we have employed the MAGPACK software.[26]

\section{Acknowledgements}


DRM, MJH and ECS acknowledge the financial support of the Spanish Governement (Grants CTQ2009-06959 and CTQ2012-32247) and the use of the infrastructure of GMMF. ECS acknowledges the support of the MCINN for a Ramon y Cajal contract and CSIC for SpLine project 25-2-779. GR would like to acknowledge financial support from the Government of India through the Department of Science and Technology (SR/S1/IC41/2010; SR/NM/NS-1119/2011) and Indian Institute of Technology, Bombay to access the high performance computing facility.

[1] J. F. Gregg, I. Petej, E. Jouguelet, C. Denis, J. Phys. D: Appl. Phys. 2002, 25, 121.

[2] S. Sanvito, Nature Mat., 2007, 6, 2007

[3] J. Camarero, E. Coronado, J. Mater. Chem., 2009, 19, 1678

[4] R. E. P. Winpenny, J. Chem. Soc., Dalton Trans., 2002, 1.

[5] E. Coronado, D. Gatteschi, J. Mater. Chem., 2006, 16, 2513.

[6] A. Pons-Balagué, S. Piligkos, S. J. Teat, J. Sánchez Costa, M. Shiddiq, S. Hill, G. Castro, P. Ferrer-Escorihuela, E. C. Sañudo Chemistry, Eur. J. 2013, 19, 9064.

[7] D. Gatteschi, A. Cornia, M. Mannini, R. Sessoli. Inorg. Chem. 2009, 48, 3408

[8] (a) M. Affronte, F. Troiani, A. Ghirri, S. Carretta, P. Santini, V. Corradini, R. Schuecker, C. Muryn, G. Timco, R. E. Winpenny, Dalton Trans. 2006, 2810; (b) A. Ghirri, V. Corradini, V. Bellini, R. Biagi, U. del Pennino, V. De Renzi, J. C. Cezar, C. A. Muryn, G. A. Timco, R. E. P. Winpenny, Marco Affronte, ACS Nano 2011, 5, 7090

[9] (a) M. Mannini, Ph. Sainctavit, R. Sessoli, Ch. Cartier dit Moulin, F. Pineider, M A. Arrio, A. Cornia, D. Gatteschi, Chem. Eur. J. 2008, 14, 7530; (b) R. Moroni, C. C. D. Moulin, G. Champion, M. A. Arrio, Ph. Sainctavit, M. Verdaguer, D Gatteschi. Phys. Rev. B 2003, 68, 064407; (c) P. Ghigna, A. Campana, A. Lascialfari, A. Caneschi, D. Gatteschi, A. Tagliaferri, F. Borgatti. Phys. Rev. B 2001, 64, 132413

[10] (a) M. Mannini, F. Pineider, P. Sainctavit, C. Danieli, E. Otero, C. Sciancalepore, A. M. Talarico, M.-A. Arrio, A. Cornia, D. Gatteschi, Roberta Sessoli. Nature Mat. 2009, 8, 194; (b) M. Mannini, F. Pineider, C. Danieli, F. Totti, L. Sorace, Ph. Sainctavit, M.-A. Arrio, E. Otero, L. Joly, J. C. Cezar, A. Cornia, R. Sessoli. Nature. 2010, 468,417

[11] D. Reta Mañeru, M. J. Heras Ojea, L. Rosado, G. Aromí, J. Zuazo, G. Castro, E. C. Sañudo. Polyhedron ICMM 2012 proceedings, 2013, in press.

[12] W. C. Hamilton, J. C. Ibers, Hydrogen Bonding in Solids. W. A. Benjamin, NY, 1968

[13] (a) J.J. McKinnon, M.A. Spackman, A.S. Mitchell, Acta Cryst. B, 2004, 60 ,627; (b) F.L. Hirshfeld, Theor. Chim. Acta, 1977, 44, 129.

[14] T. Seko, K. Ogura, Y. Kawakami, H. Sugino, H. Toyotama, J. Tanaka. Chem. Phys. Lett.,1998, 291, 438

[15] M. C. Moron, F. Palacios, J. Pons, J. Casabó, R. L. Carlin. J. Solid State and Inorg. Chem. 1991, 28, 431.
[16] S.R. Batten, P. Jensen, B. Moubaraki, K.S. Murray, R. Robson, J. Chem. Soc., Chem. Commun. 1998, 439.

[17] Introduction to Computational Chemistry; Jensen, F.; John Wiley \& Sons Ltd, 2nd Ed.

[18] Gaussian 09, Revision A.02. M. J. Frisch, G. W. Trucks, H. B. Schlegel, G. E. Scuseria, M. A. Robb, J. R. Cheeseman, G. Scalmani, V. Barone, M. Mennucci, B.; Petersson, G. A.; Nakatsuji, H.; Caricato, M.; Li, X.; Hratchian, H. P.; Izmaylov, A. F.; Bloino, J.; Zheng, G.; Sonnenberg, J. L.; Hada, M.; Ehara, K. Toyota, R. Fukuda, J. Hasegawa, M. Ishida, T. Nakajima, Y. Honda, O. Kitao, H. Nakai, T. Vreven, J. A. Montgomery, J. E. Peralta, Jr, F. Ogliaro, M. Bearpark, J. J. Heyd, E. Brothers, K. N. Kudin, V. N. Staroverov, R. Kobayashi, J. Normand, K. Raghavachari, A. Rendell, J. C. Burant, S. S. Iyengar, J. Tomasi, M. Cossi, N. Rega, J. M. Millam, M. Klene, J. E. Knox, J. B. Cross, V. Bakken, C. Adamo, J. Jaramillo, R. Gomperts, R. E.; Stratmann, O. Yazyev, A. J. Austin, R. Cammi, C. J. W. Pomelli.

[19] E. Pardo, J. Faus, M. Julve, F. Lloret. M. Carmen, J. Cano, X. Ottenwaelder, Y. Journaux, R. Carrasco, G. Blay, I. Fernandez, R. Ruiz. J. Am. Chem. Soc., 2003, 125,10770 .

[20] E. Stavitski, F. M. F. de Groot. Micron, 2010, 41, 687.

[21] (a) F. A. Cotton, D. M. L. Goodgame, M. Goodgame. J. Am. Chem. Soc. 1961, 83, 4690; (b) D. W. Herlocker, R. S. Drago. Inorg. Chem. 1968, 7, 1479; (c)S.-I. Aizawa, S. Funahashi. Inorg. Chem. 2002, 41, 4555.

[22] L. Noodleman, C. Y. Peng, D. A. Case, J. M. Mouesca. Coord.Chem. Rev. 1995 $144,199$.

[23] (a) A. D. Becke. J. Chem. Phys. 1986, 84, 4524 (b) J. P. Perdew. Phys. Rev. B. 1986, 33, 8822

[24] (a) A. Schafer, H. R. Horn, R. Ahlrichs. J. Chem. Phys. 1992, 97, 2571;(b) A Schafer, C. Huber, R. Ahlrichs. J. Chem. Phys. 1994, 100, 5829.

[25] Molekel, Advanced Interactive 3D-Graphics for Molecular Sciences; http: \www.cscs.ch/molkel/.Version 1.6(build 338), Programming Zhurko, G.A.; www.chemcraftprog.com

[26] (a) J. J. Borrás-Almenar, J. M. Clemente-Juan, E. Coronado, B. S. Tsukerblat. Inorg. Chem. 1999, 38, 6081. (b) J. J. Borrás-Almenar, J. M. Clemente-Juan, E. Coronado, B. S. Tsukerblat. J. Comput. Chem. 2001, 22, 985.

Received: ((will be filled in by the editorial staff))

Revised: ((will be filled in by the editorial staff)) Published online: ((will be filled in by the editorial staff)) 
Entry for the Table of Contents (Please choose one layout only)

\section{Layout 2:}

Molecules intact on surfaces

María José Heras Ojea, Daniel Reta Mañeru, Lidia Rosado, Juan Rubio Zuazo, German R. Castro, Subrata Tewary, Gopalan

Rajaraman, Guillem Aromí, Erika Jiménez and E. Carolina Sañudo*........... Page - Page

Characterization of a robust Co(II) fluorescent complex deposited intact on HOPG

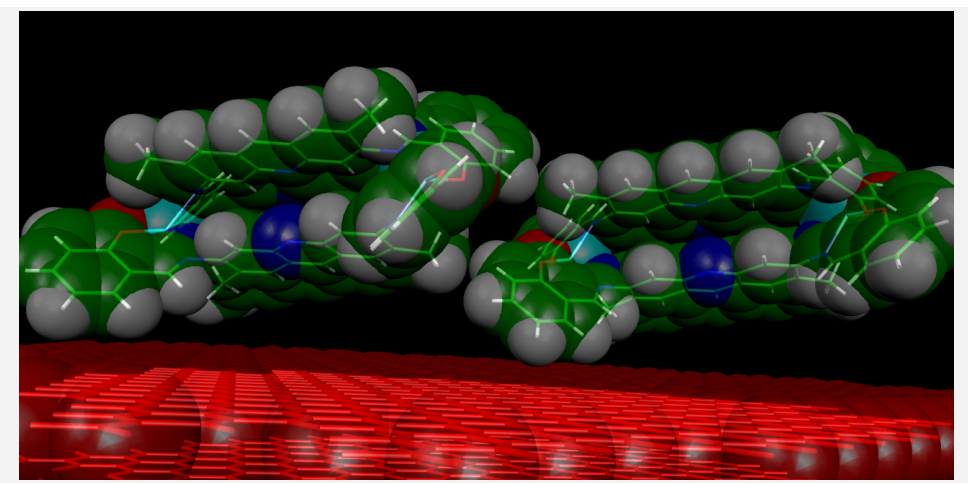

The new fluorescent ligand ACRI-1 is reported along with its graphite (HOGP) from solution. The thin films prepared have been characterized. The data show that the complex is robust and remains intact on the surface of graphite.
1) 2] (1), a fluorescent weak ferromagnet. Complex (1) has been deposited on highly ordered pyrolitic 